\title{
Effect of Solvent Quality on Stability of Diblock Copolymer Micellar Lattice
}

\author{
Hendra TAN and Hiroshi WATANABE ${ }^{\dagger}$ \\ Institute for Chemical Research, Kyoto University, Uji, Kyoto 611-0011, Japan
}

(Received April 23, 2002; Accepted August 28, 2002)

\begin{abstract}
KEY WORDS Styrene-Isoprene Diblock Copolymer / Block Copolymer Micellar Lattice / Mixed Solvent / Corona Block Correlation / Osmotic Modulus /
\end{abstract}

Diblock copolymers in a selective solvent form spherical micelles having the solvated corona and precipitated cores. $^{1-3}$ A typical example is found for polystyrene-polybutadiene (PS-PB) diblock copolymers dissolved in a PB-selective solvent, $n$-tetradecane (C14). In moderately concentrated PS-PB/C14, the micelles having the glassy PS cores and soft PB corona are further arranged on a cubic lattice. ${ }^{4-11}$

The corona blocks are required to have randomized conformations associated with a large conformational entropy. At the same time, the overlapping corona blocks are required to have mutually correlated conformations to minimize the spatial gradient of their segment concentration and reduce the osmotic free energy. Compromise of these contradicting thermodynamic requirements results in the formation of the micellar cubic lattice (having a high spatial symmetry that is helpful for this compromise). $6,8,10,11$

The micellar lattice exhibits the equilibrium modulus $G_{\mathrm{e}}$ that reflects the thermodynamic stability of the lattice: $^{4-6,8-11}$ At sufficiently large micellar concentrations, this $G_{\mathrm{e}}$ is proportional to the number density $v$ of the corona blocks. This experimental fact strongly suggests that the osmotically constrained corona blocks entropically sustain the equilibrium elasticity.

However, the measured $G_{\mathrm{e}}$ is smaller, by a factor of $\cong 10$, than the modulus expected from the classical theory of rubber elasticity, $G_{\mathrm{e}}{ }^{\circ}=\nu \mathrm{k}_{\mathrm{B}} T$ with $\mathrm{k}_{\mathrm{B}}$ and $T$ being the Boltzmann constant and absolute temperature, respectively. ${ }^{8,9}$ This difference between $G_{\mathrm{e}}$ and $G_{\mathrm{e}}{ }^{\circ}$ was firstly considered to reflect an effect of dislocations (defects) included in actual micellar lattices. However, a rheo-SANS experiment demonstrated that the shear-oriented and unoriented lattices having considerably different defect concentrations have nearly the same $G_{\mathrm{e}}$ values (with a difference $\leq 30 \%$ ). ${ }^{9}$ Thus,

${ }^{\dagger}$ To whom correspondence should be addressed. the defects have just a secondary effect on the $G_{\mathrm{e}}$ value (at high micellar concentrations).

On the basis of this experimental fact, the difference between the measured $G_{\mathrm{e}}$ and calculated $G_{\mathrm{e}}{ }^{\circ}$ was speculated to reflect the osmotically induced correlation of the overlapping corona blocks: ${ }^{9}$ This correlation, the key factor in the micellar lattice formation, does not allow individual corona blocks to behave as independent entropic strands. Instead, some number $(\beta)$ of the correlated corona blocks should together behave as an independent strand, thereby reducing the effective strand number density by the factor of $\beta$. The difference between the $G_{\mathrm{e}}$ and $G_{\mathrm{e}}{ }^{\circ}$ values possibly results from this reduction.

This hypothesis suggests that the $G_{\mathrm{e}}$ value changes with the magnitude of the osmotic correlation. If this correlation is fully screened, the driving force of the micellar lattice formation vanishes and the micelles cannot sustain the equilibrium elasticity. This is the case for the PS-PB micelles in a matrix of low molecular weight homopolybutadiene (that is chemically identical to the PB corona blocks to achieve this screening). ${ }^{6,8}$ However, the equilibrium elasticity is expected to be enhanced if the osmotic correlation is weakened just moderately.

On the basis of this expectation, we have attempted to examine an effect of the magnitude of the osmotic correlation on the equilibrium modulus $G_{\mathrm{e}}$. This magnitude changes with several thermodynamic parameters, and a change with a solvent quality is rather well understood, as explained below.

A gradient in the concentration $(C)$ profile is suppressed and the osmotic correlation of overlapping chains is enhanced with increasing osmotic modulus, $C^{-1}(\partial \Pi / \partial C)$ with $\Pi$ being the osmotic pressure. The $\Pi$ data are available for semidilute solutions of homopolystyrene (hPS) chains in good and theta solvents, 
toluene at $25^{\circ} \mathrm{C}^{12}$ and cyclohexane at $35^{\circ} \mathrm{C} .{ }^{13}$ From these data (obeying the scaling molecular picture, ${ }^{14}$ ) an empirical equation for the osmotic modulus is obtained as $^{12,13}$

$$
\begin{aligned}
& \frac{1}{C} \frac{\partial \Pi}{\partial C}=1.14 \times 10^{8} C^{0.274} \text { in toluene }\left(25^{\circ} \mathrm{C}\right) \\
& \frac{1}{C} \frac{\partial \Pi}{\partial C}=1.7 \times 10^{7} C^{1.0} \text { in cyclohexane }\left(35^{\circ} \mathrm{C}\right)
\end{aligned}
$$

As noted from eqs 1 and 2 (both in cgs unit), the osmotic modulus is smaller and the osmotic correlation is weaker in the theta solvent than in the good solvent. (The $C$ dependence of the osmotic modulus is stronger in the theta solvent but the numerical prefactor is much larger in the good solvent, thereby giving the smaller osmotic modulus in the former.) More generally, we can confirm a continuous decrease of the osmotic modulus with decreasing solvent quality for the $\mathrm{hPS} /$ cyclohexane solutions at various temperatures. ${ }^{13}$

Considering this relationship between the solvent quality and osmotic modulus, we utilized mixtures of n-tetradecane (C14) and diethyl phthalate (DEP) as the selective solvent for polyisoprene (PI) block in PS-PI copolymers to tune the solvent quality for the PI block by changing the C14/DEP mixing ratio: $\mathrm{C} 14$ is a good solvent for PI and a non-solvent for PS, while DEP is a non-solvent for PI and a marginal solvent for PS. In all PS-PI/C14/DEP solutions examined, the DEP content was kept small $(\leq 7 \mathrm{wt} \%)$ so that the spherical micelles having PS cores and PI corona were formed. The equilibrium modulus $G_{\mathrm{e}}$ was measured for these solutions, and an effect of the solvent quality on $G_{\mathrm{e}}$ was examined. It turned out that $G_{\mathrm{e}}$ changes with the solvent quality in a way consistent with the above expectation. These results are presented in this article.

\section{EXPERIMENTAL}

\section{Materials}

PS-PI diblock copolymers were anionically polymerized with sec-butyllithium (initiator) in benzene. These copolymer samples were characterized with GPC (CO8020 and DP-8020, Tosoh) equipped with refractive index (RI) and ultraviolet absorption (UV) monitors (LS-8000 and UV-8020, Tosoh) connected in series. Monodisperse polystyrenes (Tosoh TSK's) were utilized as elution standards. The molecular characteristics thus determined are summarized in Table I.

The systems subjected to viscoelastic measurements were the copolymer solutions in mixtures of C14 (Wako) and DEP (Wako). These solutions were prepared by firstly dissolving prescribed masses of the copolymer, C14, and DEP in excess methylene chloride (MC) and then allowing MC to thoroughly evaporate.
Table I. Characteristics of PS-PI Diblock Copolymers

\begin{tabular}{cccc}
\hline \multicolumn{1}{c}{ Code $^{\mathrm{a}}$} & $10^{-3} M_{\mathrm{S}}$ & $10^{-3} M_{\mathrm{I}}$ & $M_{\mathrm{w}} / M_{\mathrm{n}}$ \\
\hline PS-PI 8.6-43 & 8.6 & 43 & 1.04 \\
PS-PI 43-86 & 43.2 & 85.8 & 1.06 \\
PS-PI 68-122 & 68.6 & 122 & 1.05 \\
PS-PI 111-180 & 111 & 180 & 1.07 \\
\hline \multicolumn{2}{c}{${ }^{\mathrm{a}}$ Code number indicates $10^{-3} M_{\mathrm{S}}-10^{-3} M_{\mathrm{I}}}$.
\end{tabular}

C14 is a good solvent for PI and a non-solvent for PS, while DEP is a non-solvent for PI and a marginal solvent for PS. In the PS-PI/C14/DEP solutions examined, the DEP content $w_{\text {DEP }}$ in the mixed solvent was kept small $(\leq 7 \mathrm{wt} \%)$ and the copolymer concentration $C_{\text {PS-PI }}$ was chosen to be moderately large $\left(C_{\mathrm{PS}-\mathrm{PI}}=15 \mathrm{wt} \%\right.$ for the SI $8.6-43$ and SI $43-86 \mathrm{sam}-$ ples and $C_{\mathrm{PS}-\mathrm{PI}}=25 \mathrm{wt} \%$ for the SI $68-122$ and SI 111-180 samples). Under these conditions, the spherical micelles with PS cores (having the volume fraction $<0.11$ ) and PI corona should have been formed in the solutions, ${ }^{15,16}$ as suggested from a preliminary solubility test for hPS.

To examine the solubility of the mixed solvent toward the PI block, we measured the intrinsic viscosity $[\eta]_{\text {PI }}$ of a high-M homo-PI $\left(M_{\mathrm{w}}=180 \times 10^{3}\right.$ and $\left.M_{\mathrm{w}} / M_{\mathrm{n}}=1.06\right)$ at $10^{\circ} \mathrm{C}$, the temperature for the viscoelastic measurements for the PS-PI solutions. The results were: $[\eta]_{\mathrm{PI}}=99 \mathrm{~g}^{-1} \mathrm{~cm}^{3}$ in $\mathrm{C} 14$ and $[\eta]_{\mathrm{PI}}=$ $94 \mathrm{~g}^{-1} \mathrm{~cm}^{3}$ in the C14/DPE mixed solvent with $w_{\text {DEP }}=$ $7 \mathrm{wt} \%$. (The $[\eta]_{\mathrm{PI}}$ value in $\mathrm{C} 14$ is close to the value $103 \mathrm{~g}^{-1} \mathrm{~cm}^{3}$ in heptane ${ }^{17}$ chemically similar to $\mathrm{C} 14$.) Thus, $[\eta]_{\text {PI }}$ decreases by $5 \%$ with increasing $w_{\text {DEP }}$ up to $7 \mathrm{wt} \%$, suggesting a moderate decrease of the solubility and the corresponding reduction in the osmotic correlation with $w_{\text {DEP }}$. (The $[\eta]_{\text {PI }}$ value in the mixed solvent $\left(w_{\text {DEP }}=7 \mathrm{wt} \%\right)$ is well above the value in dioxane at $34{ }^{\circ} \mathrm{C}(\Theta$ temperature $), 61.5 \mathrm{~g}^{-1} \mathrm{~cm}^{3}$ for $M=180$ $\times 10^{3},{ }^{18}$ meaning that the mixed solvent is still classified as a marginal solvent for PI.)

\section{Linear Viscoelastic Measurements}

Dynamic viscoelastic measurements were carried out at $10{ }^{\circ} \mathrm{C}$ with a stress-control rheometer (SR-5000; Rheometrics) with a parallel plate fixture having a diameter of $25.0 \mathrm{~mm}$ and a gap width of $1 \mathrm{~mm}$. The PS$\mathrm{PI} / \mathrm{C} 14 / \mathrm{DEP}$ solutions were charged in the gap and disordered at $70-90{ }^{\circ} \mathrm{C}$ for $15 \mathrm{~min}$ and quiescently ordered (without being deformed) by cooling to the test temperature. This process enabled equilibration of micellar lattice by erasing undesirable shear history during the sample loading. After annealing at $10^{\circ} \mathrm{C}$ for $12 \mathrm{~h}$, the storage and loss moduli, $G^{\prime}$ and $G^{\prime \prime}$, were measured. The applied stress level was chosen in a way that the oscillatory strain amplitude $\gamma_{0}$ did not exceed 0.01 , and 

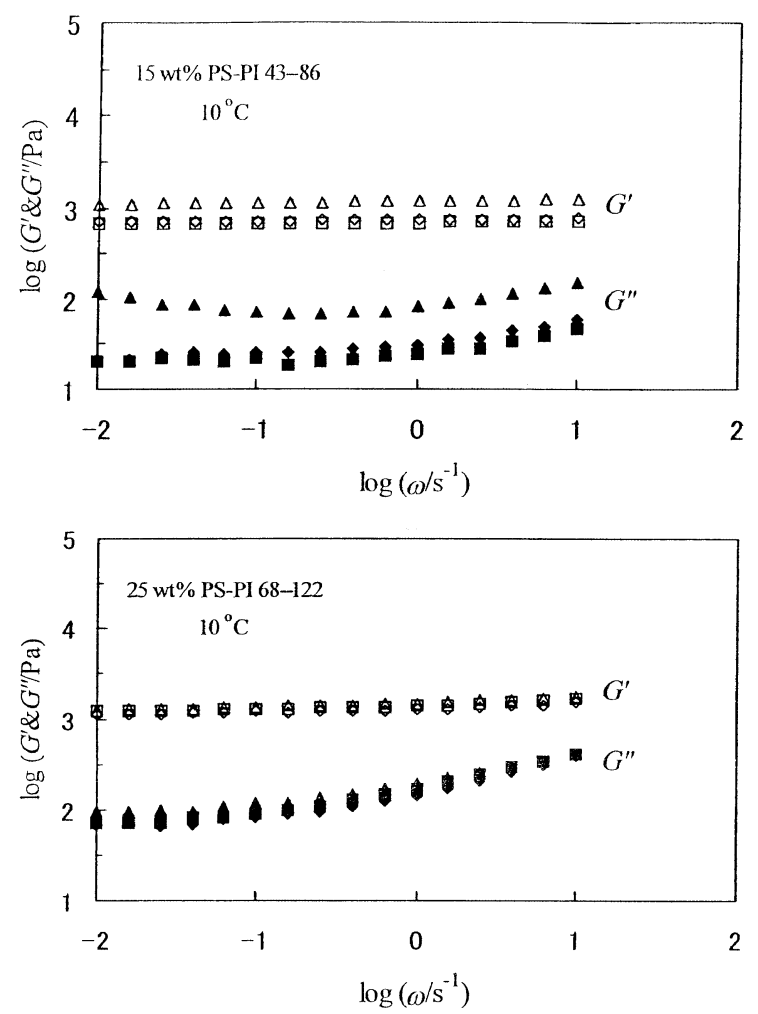

Figure 1. Linear viscoelastic behavior of the solutions of PSPI 43-86 (15 wt\%; top panel) and PS-PI 68-122 (25wt\%; bottom panel) in mixed C14/DEP solvent. Unfilled and filled symbols indicate the storage and loss moduli, $G^{\prime}$ and $G^{\prime \prime}$. Squares, diamonds, and triangles, respectively, represent the data obtained in the solvent with the DEP content $w_{\text {DEP }}=0,4$, and $7 \mathrm{wt} \%$.

the linearity of the viscoelastic response was confirmed by changing the stress level (while keeping $\gamma_{0}<0.01$ ). The measurement for each solution was carried out at least twice from the scratch to ensure the repeatability of the data.

\section{RESULTS AND DISCUSSION}

Figure 1 shows the representative $G^{\prime}$ and $G^{\prime \prime}$ data obtained for the SI 43-86 and SI 68-122 solutions in the mixed C14/DEP solvent at $10{ }^{\circ} \mathrm{C}$. The DEP content $w_{\text {DEP }}$ in the solvent is varied from $0 \mathrm{wt} \%$ (pure $\mathrm{C} 14$ ) to $7 \mathrm{wt} \%$. In the entire range of the angular frequency $\omega\left(\right.$ down to $10^{-2} \mathrm{~s}^{-1}$ ), the solutions exhibit highly elastic behavior characterized with the $\omega$-insensitive $G^{\prime}$ and much smaller $G^{\prime \prime}$. This behavior reflects the elasticity of the lattice of the PS-PI micelles (with glassy PS cores and solvated PI corona) formed therein. We utilized the $G^{\prime}$ data at the lowest $\omega\left(=10^{-2} \mathrm{~s}^{-1}\right)$ as the equilibrium modulus $G_{\mathrm{e}}$ of the micellar lattice.

In Figure 2, the $G_{\mathrm{e}}$ thus obtained for respective PS-PI systems is plotted against $w_{\text {DEP }}$. The changes of $G_{\mathrm{e}}$ with $w_{\text {DEP }}$ can be classified in two groups: For the PS-PI 43-86 and PS-PI 111-180 systems, $G_{\mathrm{e}}$ increases with increasing $w_{\text {DEP }}$ up to $7 \mathrm{wt} \%$; namely, the equilib-

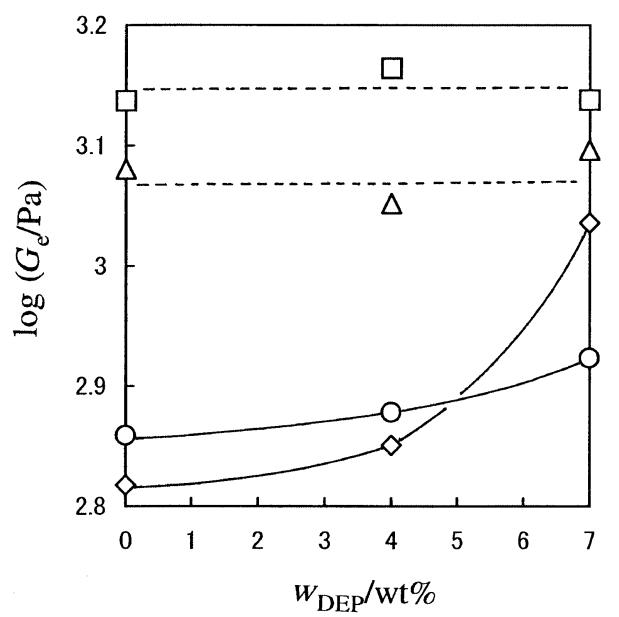

Figure 2. Dependence of the equilibrium modulus $G_{\mathrm{e}}$ of the PS-PI solutions on the DEP content $w_{\text {DEP }}$ in the mixed C14/DEP solvent. square: $15 \mathrm{wt} \%$ PS-PI $8.6-43$, triangle: $25 \mathrm{wt} \%$ PS-PI $68-$ 122 , circle $25 \mathrm{wt} \%$ PS-PI $111-180$, diamond: $15 \mathrm{wt} \%$ PS-PI $43-86$.

rium elasticity of the lattice is enhanced as the solvent quality is reduced. On the other hand, $G_{\mathrm{e}}$ is insensitive to $w_{\text {DEP }}$ for the PS-PI $8.6-43$ and PS-PI $68-122$ systems.

Some argument is necessary for these $w_{\text {DEP }}$ dependencies of $G_{\mathrm{e}}$. So far, we have been focusing on only one effect of mixing C14 and DEP, the reduction of the osmotic correlation of overlapping corona (PI) blocks on a decrease of the solvent quality. This effect leads to the increase of $G_{\mathrm{e}}$ with increasing $w_{\text {DEP }}$, as explained earlier. However, three other effects competing with the first effect could arise on mixing of C14 and DEP, as explained below.

Since DEP is a marginal solvent for the PS block, the PS cores may be plasticized in presence of DEP. If the glass transition point reduced by DEP reaches the measuring temperature, $10^{\circ} \mathrm{C}$, the PS-PI junction becomes mobile. The junction mobility considerably destabilizes the micellar lattice thereby reducing the lattice elasticity, as noted previously. ${ }^{5}$

If the PS cores are considerably swollen, the PS-PI junction density on the core surface would be reduced and $G_{\mathrm{e}}$ could decrease accordingly. Although we do not expect a significant swelling of the $\mathrm{S}$ cores in the solvents with $w_{\text {DEP }} \leq 7 \mathrm{wt} \%$, we can have this swelling in our mind as a potential factor affecting $G_{\mathrm{e}}$.

If the PI corona blocks take expanded conformation in pure $\mathrm{C} 14$ due to the excluded volume (EV) interaction, the blocks would shrink on mixing of $\mathrm{C} 14$ and DEP (non-solvent for PI) to reduce their mutual overlapping. This reduction of overlapping, if occurring significantly, could destabilize the micellar lattice and decrease $G_{\mathrm{e}}$. The EV interaction should not be significant for the PI blocks in the rather concentrated solutions examined in this study. However, we may regard 
this corona shrinkage as the other potential factor affecting $G_{\mathrm{e}}$.

Thus, all of the second to fourth effects of DEP (junction mobility, core swelling, and corona shrinkage) tend to decrease $G_{\mathrm{e}}$ on an increase of $w_{\text {DEP }}$. Considering this point, we may judge from the data of Figure 2 that the first effect (reduction of the osmotic correlation) is never overwhelmed by the other effects in the PS-PI systems thereby giving the $G_{\mathrm{e}}$ that never decreases with increasing $w_{\text {DEP }}$ up to $7 \mathrm{wt} \%$. In other words, these data strongly suggest that the mild reduction of the osmotic correlation itself enhances the micellar lattice stability/equilibrium elasticity. This result is consistent with the hypothesis about the difference between the measured $G_{\mathrm{e}}$ and calculated $G_{\mathrm{e}}{ }^{\circ}$ explained earlier.

\section{CONCLUSION}

The stability of the lattice of the PS-PI diblock copolymer micelles, measured as the equilibrium modulus, never decreases on a small increase of the content of DEP (non-solvent for the PI corona) in the solvent. This result strongly suggests that that the mild reduction of the osmotic correlation due to this small increase enhances the lattice stability.

Acknowledgment. H. T. gratefully acknowledges the Jinnai scholarship provided from Association of International Education, Japan.

\section{REFERENCES}

1. T. Kotaka, T. Tanaka, and H. Inagaki, Polym. J., 3, 327 (1972).

2. T. Kotaka and J. L. White, Trans. Soc. Rheol., 17, 587 (1973).

3. T. Kotaka, T. Tanaka, M. Hattori, and H. Inagaki, Macromolecules, 11, 138 (1978).

4. H. Watanabe, T. Kotaka, T. Hashimoto, M. Shibayama, and H. Kawai, J. Rheol., 26, 153 (1982).

5. H. Watanabe and T. Kotaka, Polym. J., 15, 337 (1983).

6. H. Watanabe and T. Kotaka, J. Rheol., 27, 223 (1983).

7. H. Watanabe, J. Soc. Rheol. Jpn., 22, 193 (1994).

8. H. Watanabe, Acta Polym., 48, 215 (1997).

9. H. Watanabe, T. Kanaya, and Y. Takahashi, Macromolecules, 34, 662 (2001).

10. H. Watanabe, Kobunshi Ronbunshu, 58, 135 (2001).

11. H. Watanabe, "Structure and Properties of Multiphase Polymeric Materials", in "Structure and Properties of Multiphase Polymeric Materials", T. Araki, Q. Tran-Cong, and M. Shibayama, Ed., Marcel Dekker Inc., New York, N.Y., 1998, p 317.

12. I. Noda, Y. Higo, N. Ueno, and T. Fujimoto, Macromolecules, 17, 1055 (1984).

13. P. Stepanek, R. Perzynski, M. Delsanti, and M. Adam, Macromolecules, 17, 2340 (1984).

14. P. G. de Gennes, "Scaling Concepts in Polymer Physics", Cornell University Press, Ithaca, N.Y., 1979.

15. H. Watanabe and T. Kotaka, Macromolecules, 16, 769 (1983).

16. H. Watanabe and T. Kotaka, Macromolecules, 17, 342 (1984).

17. O. Urakawa, K. Adachi, and T. Kotaka, Macromolecules, 26, 2036 (1993).

18. M. Kurata and Y. Tsunashima, "Viscosity-Molecular Weight Relationships and Unperturbed Dimensions of Linear Chain Molecules", in "Polymer Handbook 3rd ed", E. H. 1mmergut, Ed., John Wiley \& Sons, lnc, New York, N.Y., 1989, p VII-1. 